\title{
Metal-Insulator Transition in Zinc-Doped LaSrCuO
}

\author{
A. Malinowski, M.Z. Cieplak, M. Berkowski, \\ W. Plesiewicz AND T. SKOŚKIEWICZ \\ Institute of Physics, Polish Academy of Sciences \\ al. Lotników 32/46, 02-668 Warsaw, Poland
}

The magnetotransport in the vicinity of the metal-insulator transition in $\mathrm{La}_{1.85} \mathrm{Sr}_{0.15} \mathrm{Cu}_{x} \mathrm{Zn}_{1-x} \mathrm{O}_{4}$ is studied in the $\mathrm{mK}$ temperature range. Both longitudinal and transverse magnetoresistance are negative indicating the importance of spin effects. The magnitude of transverse magnetoresistance is larger than the magnitude of longitudinal magnetoresistance, indicating the absence of positive orbital magnetoresistance, in sharp contrast to strongly underdoped $\mathrm{La}_{2-x} \mathrm{Sr}_{x} \mathrm{CuO}_{4}$. Both transverse and longitudinal magnetoresistance are proportional to the relative change of zero-field conductivity. This suggests that low-temperature localization of carriers may originate in the spin-disorder scattering on the spin droplets around Zn-impurities.

PACS numbers: 74.72.-h, 71.30.+h, 74.25.Fy

\section{Introduction}

Superconductivity in the high- $T_{\mathrm{C}}$ cuprates occurs in the vicinity of the metal-insulator transition (MIT). While the overdoped range of carrier concentration is believed to be a normal metal, well-described by Fermi-liquid theory, specimens from the underdoped region show some features of insulating-like behavior. In particular, these features are visible in the electric transport at low temperatures. When temperature is decreased the resistivity first decreases linearly with decreasing $T$ displaying metallic-like character, reaches a minimum and finally starts to increase. The location of the minimum shifts toward higher $T$ with decreasing carrier concentration. Such a behavior was seen both in the holeand electron-doped cuprates. The nature of the localization effects in these two cases is a subject of debate. Magnetoresistance (MR) measurements allow for a deeper insight into the physics of these phenomena.

At low temperatures a negative MR in the normal state is observed. In recent experiments on $\mathrm{PrCeCuO}$ it has been found that most of this negative MR comes from an isotropic spin effect, and not from an anisotropic orbital effect [1]. 
The temperature, at which a negative longitudinal MR appears, coincides approximately with the temperature of the upturn in zero-field resistivity. Therefore, it has been suggested that the resistivity upturn in electron-doped cuprates was suggested to be due to localization effects induced by spin-scattering [1]. On the other hand, recently we have observed a positive orbital magnetoresistance in the strongly underdoped $\mathrm{LaSrCuO}$. This suggests the importance of interaction effects for the localization of carriers in hole-doped cuprates [2].

In the present study we examine the magnetotransport in the vicinity of the MIT induced by the zinc doping in $\mathrm{La}_{1.85} \mathrm{Sr}_{0.15} \mathrm{Cu}_{x} \mathrm{Zn}_{1-x} \mathrm{O}_{4}$. It is known that the doping leaves carrier concentration unaffected, leading to a metallic, nonsuperconducting phase for $x$ between 0.08 and 0.1 , followed by MIT which occurs at $x=0.14$ [3]. We observe a negative longitudinal magnetoresistance (LMR) indicating the importance of spin effects. The analysis of both transverse magnetoresistance (TMR) and LMR shows that transport data are consistent with the picture of spin-disorder scattering of carriers on spin droplets around Zn-impurities.

\section{Results and discussion}

We study the in-plane resistivity of the $\mathrm{La}_{1.85} \mathrm{Sr}_{0.15} \mathrm{Cu}_{x} \mathrm{Zn}_{1-x} \mathrm{O}_{4}$ films in the vicinity of MIT, with the $\mathrm{Zn}$ concentration $x=0.08,0.10$, and 0.12 . The $c$-axis oriented epitaxial films are made by pulsed laser deposition method on the $\mathrm{SrLaAlO}_{4}$ substrate. In the zero-field measurements we are able to achieve $20 \mathrm{mK}$ while edge-currents during field sweeps limit our lowest temperature for MR measurements to $45 \mathrm{mK}$. The magnetic field up to $9 \mathrm{~T}$ is applied both parallelly and perpendicularly to $\mathrm{CuO}_{2}$ planes.

Below a broad maximum at around $35 \mathrm{~K}$ zero-field conductivity for all films starts to decrease with temperature, approximately as $\ln T$. A careful examination shows that $\sigma$ vs. $T$ dependence below $\sim 20 \mathrm{~K}$ may be well approximated as

$$
\sigma=\sigma_{0 \mathrm{~K}}+C \ln \left(\left(T+T_{0}\right) / T_{0}\right) .
$$

The best fits, shown in Fig. 1 as the solid lines, give $T_{0}=0.48 \mathrm{~K}, 0.36 \mathrm{~K}$, and $0.28 \mathrm{~K}$ for $x=0.08,0.10$, and 0.12 , respectively. The ratio $C / \sigma_{0 \mathrm{~K}}$ appears to be the same and equals to 0.09 for samples with $k_{\mathrm{F}} l>1$ (where $k_{\mathrm{F}}$ is the Fermi wave vector and $l$ - mean free path), i.e. with $x=0.08$ and 0.10 . On the other hand, $C / \sigma_{0 \mathrm{~K}}=0.18$ for $x=0.12$.

The LMR, defined as $[\rho(B)-\rho(0)] / \rho(0)$, where $\rho(B)$ is measured in the field applied parallel to $\mathrm{CuO}_{2}$ planes, becomes negative below about $2 \mathrm{~K}$. In addition, its magnitude grows with decreasing $T$ and increasing $x$. For $x=0.12$ LMR achieves $-5 \%$ in the field of $8 \mathrm{~T}$ at $T=45 \mathrm{mK}$. Such a large negative LMR strongly suggests spin-related effects. Down to about $500 \mathrm{mK}$ the LMR changes almost linearly with the magnetic field. As illustrated in Fig. 2 for the sample with $x=0.10$, the LMR scales as $B / T$ in the $T$ range from $0.5 \mathrm{~K}$ to $2 \mathrm{~K}$. The scaling breaks down below $\sim 500 \mathrm{mK}$. This is caused by the saturation of LMR at lower $T$, resembling somewhat the behavior which occurs in the Kondo systems. 


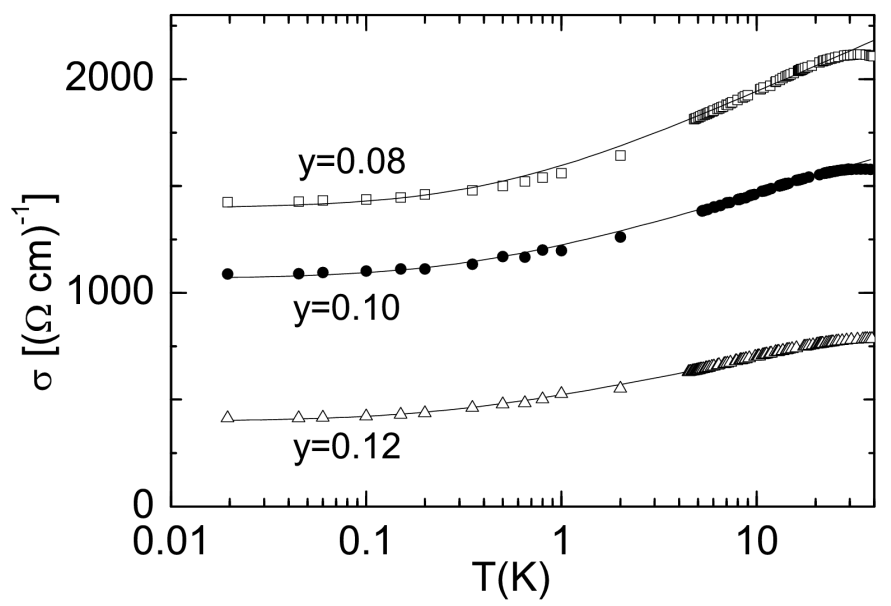

Fig. 1. The temperature dependence of the zero-field conductivity for three samples with $x=0.08,0.10$, and 0.12 . The solid lines are the best fits to Eq. (1).

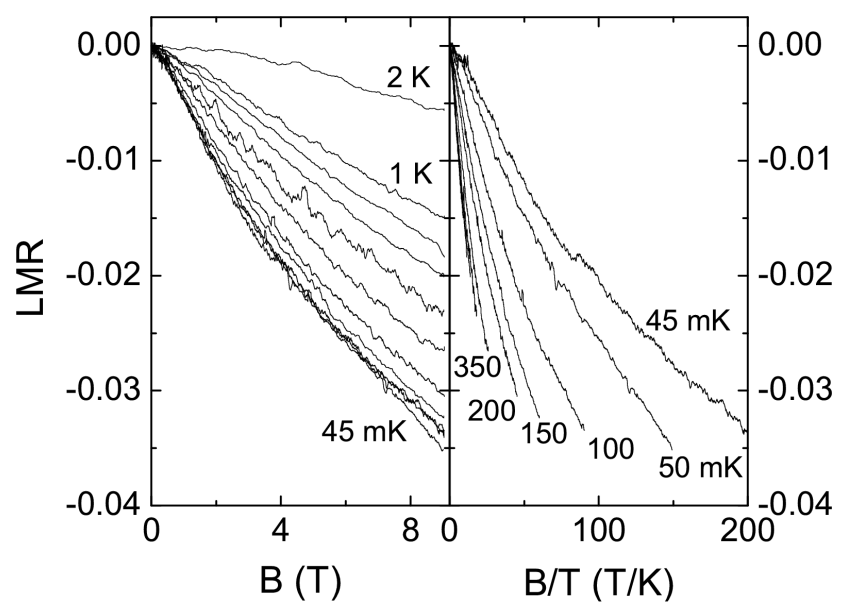

Fig. 2. Left panel: the field dependence of longitudinal magnetoresistivity for the sample with $x=0.10$ at different temperatures, from top to bottom: $2 \mathrm{~K}, 1 \mathrm{~K}$, and 800 , $650,500,350,200,150,100,50$, and $45 \mathrm{mK}$. Right panel: the same data plotted as a function of $B / T$.

TMR is also negative and its magnitude is larger than the magnitude of LMR. This indicates the absence of positive orbital magnetoresistance (OMR), defined as a difference TMR-LMR. The positive OMR has been observed previously for strongly underdoped $\mathrm{LaSrCuO}$ and interpreted as an evidence of strong electronelectron interactions [2]. Anisotropy, defined as the ratio TMR/LMR for $B=8 \mathrm{~T}$, saturates at the value of 1.6, independent from $\mathrm{Zn}$ content, as $T$ decreases below 300 mK. 
The negative difference TMR-LMR cannot be ascribed to the weak localization effects. In conventional quantum theories for two-dimensional metals negative OMR should be proportional to $\ln B$. For $\mathrm{La}_{1.85} \mathrm{Sr}_{0.15} \mathrm{Cu}_{x} \mathrm{Zn}_{1-x} \mathrm{O}_{4}$ the difference TMR-LMR changes linearly with the field.

Looking for the possible origins of localization effects manifesting themselves by the saturation of the zero-field $\sigma$, and for the explanation of the negative MR we compare both quantities ( $\sigma$ and MR) at the same $T$. In Fig. 3 we plot LMR, i.e. the relative change of resistivity at the magnetic field $B=8 \mathrm{~T}$ as a function of the relative change of conductivity with temperature at zero field, $\Delta \sigma / \sigma_{0 \mathrm{~K}}=$ $\left(\sigma_{T}-\sigma_{0 \mathrm{~K}}\right) / \sigma_{0 \mathrm{~K}}$. Both quantities are related by linear dependence: LMR $=$ $\operatorname{LMR}(T=0 \mathrm{~K})+A \Delta \sigma / \sigma_{0 \mathrm{~K}}$, which means that larger (relative) localization corresponds to a larger field effect suggesting a common origin of both effects. The coefficient $A$ is identical and equal to 0.17 for $x=0.08$ and $x=0.10$, and equal to 0.14 for $x=0.12$. For transverse configuration results are similar - TMR also changes linearly $\Delta \sigma / \sigma_{0 \mathrm{~K}}$, although with different values of $A$.

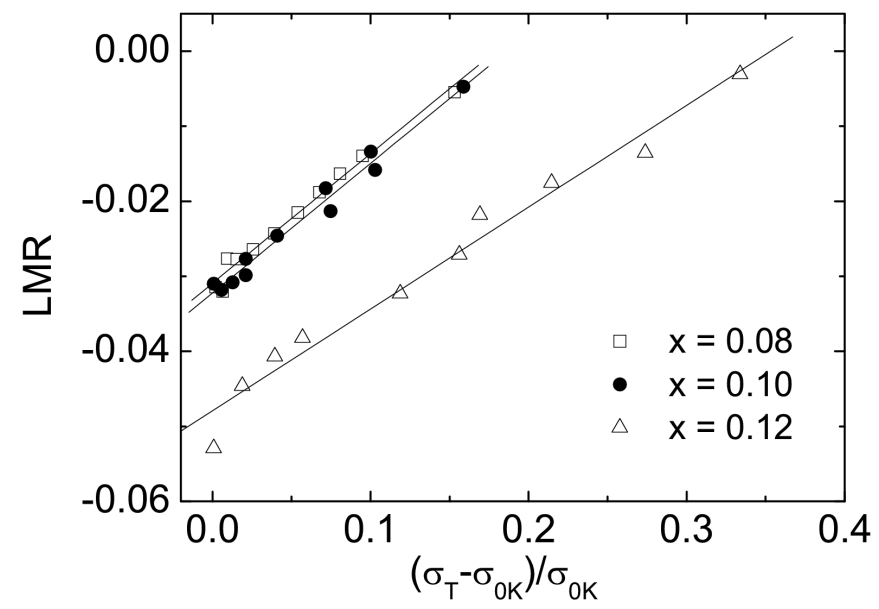

Fig. 3. Longitudinal magnetoresistivity, LMR, as a function of $\left(\sigma_{T}-\sigma_{0 \mathrm{~K}}\right) / \sigma_{0 \mathrm{~K}}$. The temperature of the measurement, $T$, is the implicit parameter. The solid lines are linear fits, as described in the text.

A model-independent analysis of ${ }^{63} \mathrm{Cu}$ NMR spectra for another cuprate, $\mathrm{YBa}_{2} \mathrm{Cu}_{3} \mathrm{O}_{6.7}$, revealed that $\mathrm{Zn}$ impurity enhances antiferromagnetic (AF) correlations in $\mathrm{CuO}_{2}$ planes inducing staggered moments on many $\mathrm{Cu}$ sites around a single Zn ion [4]. If so, many such "spin droplets" may cause localization of charge carriers at low $T$ and when applied field destroys AF correlations the rate of spin-disorder scattering decreases leading to negative MR. However, a more detailed quantitative model is needed.

In conclusion the magnetotransport properties observed for impurity-doped $\mathrm{LaSrCuO}$ in the vicinity of metal-insulator transition are very different from these 
observed for hole-doped LaSrCuO. They are consistent with the model of spin droplets around Zn-impurities inducing charge localization.

\section{Acknowledgment}

This work was supported by the State Committee for Scientific Research grant P2 P03B 04423.

\section{References}

[1] Y. Dagan, M.C. Barr, W.M. Fisher, R. Beck, T. Dhakal, A. Biswas, R.L. Greene, Phys. Rev. Lett. 94, 057005 (2005).

[2] Marta Z. Cieplak, A. Malinowski, S. Guha, M. Berkowski, Phys. Rev. Lett. 92, 187003 (2004).

[3] K. Karpińska, Marta Z. Cieplak, S. Guha, A. Malinowski, T. Skośkiewicz, W. Plesiewicz, M. Berkowski, B. Boyce, T.R. Lemberger, P. Lindenfeld, Phys. Rev. Lett. 84, 155 (2000).

[4] M.-H. Julien, T. Fehér, M. Horvatić, C. Berthier, O.N. Bakharev, P. Ségransan, G. Collin, J.-F. Marucco, Phys. Rev. Lett. 84, 3422 (2000). 